\title{
LA CONTINUIDAD PEDAGÓGICA EN EDUCACIÓN SUPE- RIOR EN TIEMPOS DE LA PANDEMIA
}

\section{THE PEDAGOGICAL CONTINUITY APPLIED TO HIGHER EDUCATION IN TIMES OF THE PANDEMIC} \author{
Ponce de León ${ }^{3}$ \\ 1. ${ }^{*}$ Docente Universidad Autónoma de Chihuahua, Chihuahua, México. \\ Email: mcossio@uach.mx (D) https://orcid.org/0000-0001-6511-6547 \\ 2. Docente Universidad Autónoma de Chihuahua, Chihuahua, México. \\ Email: oquinones@uach.mx (D) https://orcid.org/0000-0002-0109-9159 \\ 3. Docente Universidad Autónoma de Chihuahua, Chihuahua, México. \\ Email: acossio@uach.mx (D) https://orcid.org/0000-0001-6223-4337.
}

Dra. Mariana Cossio Ponce de León ${ }^{1}$, M.T.B. Osiris Quiñones Domínguez ${ }^{2}$, M.C. Alejandra Cossio

Como Citar: Cossío Ponce de León, M., Quiñones Domínguez, O., \& Cossio Ponce de León, A. (2020). La Continuidad pedagógica en educación superior en tiempos de la pandemia. Revista Publicando, 8(28), 67-73. https://doi.org/10.51528/rp.vol8.id2159

\section{RESUMEN:}

De frente a la exigencia de trasladar la enseñanza presencial a enseñanza virtual, con la aparición de la COVID-19 y con ello se experimentó el distanciamiento social, ajustes de calendario y capacitación exprés, para enfrentar el paradigma educativo que la modalidad a distancia impone. La comunidad universitaria participó en este reto institucional y una vez concluido el proceso creímos importante caracterizar la capacidad para trabajar en línea de los estudiantes. Estos son resultados preliminares del proyecto "Continuidad pedagógica en Educación Superior en tiempo de la pandemia". Se diseñó un estudio descriptivo y retrospectivo, aplicamos una encuesta en línea la última semana del ciclo escolar. La muestra se conformó por 129 universitarios, 32.6\% (42) masculinos y $67.4 \%$ (87) femeninos de nivel Licenciatura. La edad promedio de los estudiantes fue $21.01+4.7$, el $29.5 \%$, contaron con conexión a internet suficiente y de calidad para realizar el trabajo en la plataforma, el $53.5 \%$ con conexión regular y el $17 \%$ sin conexión. El $80 \%$ de los estudiantes adaptaron un espacio para desarrollar actividades académicas. Los estudiantes manifiestan una evaluación complicada en el $76 \%$, el trabajo fue excesivo en el $85.3 \%$ y el $53.6 \%$ piensa que la calificación del curso no corresponde al trabajo y dedicación que tuvieron. Los estudiantes dijeron en el $78.3 \%$ que su calificación no representa el conocimiento adquirido. Se encontraron diferencias significativas, la percepción de las mujeres de mayor facilidad en tomar clases en línea y los varones prefieren esperar a que todo se normalice para continuar sus estudios.

\section{Palabras Clave:}

Alternativas pedagógicas, educación superior, educación virtual, adaptación escolar.

\section{ABSRTACT:}

With the appearance of COVID-19 and concepts like social distancing, rescheduling and express training where essential to tackle the paradigm that this new distance-based model imposed. The whole of the universe's community was needed to participate in this institutional challenge and, once the process had ended, we deemed important to test the capabilities of our students to work online. These are the preliminary results in the project "Pedagogical Continuity applied to a higher education in times of pandemic". First, descriptive, and retrospective studies were designed, later to be applied in the form of an online test on the last week of academic term. The test had as subject a total of 129 university students, of which $32.6 \%$ (42) where male and $67.4 \%$ (87) female, all of them undergraduates. The average age of the testers was $21.01+4.7$. Of these $29.5 \%$ counted with a reliable internet connection to perform their duties, $53.5 \%$ had faulty irregular connections and the $17 \%$ had no connection at all. $80 \%$ of students admitted to have created a workplace within their homes to better perform their school work. Students manifested that in $78.3 \%$ of cases the evaluation felt complex, $85.3 \%$ felt they were overworked, while the remaining $53.6 \%$ feel that the grade they were given was not on-par with the time an effort they gave. $78.3 \%$ of students said that their grades were not a one-on-one representation of their acquired knowledge.

\section{KEYWORDS:}

Pedagogical alternatives, higher education, virtual education, school adaptation. 


\section{INTRODUCCIÓN}

$\mathrm{L}$ a aparición de la COVID-19 en nuestro contexto ha provocado importantes ajustes, ha representado por los riesgos que implica un confinamiento obligatorio para todos, sin precedentes. Esto nos llevó en el ámbito educativo del aula presencial al ambiente virtual. Este es un hecho histórico en el que las instituciones educativas de todos los niveles han tenido que colaborar de manera estrecha con los diferentes órdenes de gobierno buscando contribuir en las estrategias planteadas que permitan la protección de la salud de la comunidad académica (estudiantes, empleados administrativos, docentes y autoridades) pero además permitan concluir el ciclo escolar.

Bajo esta emergencia sanitaria se ha tenido que dar paso a prácticas pedagógicas de carácter también emergente; aunque si bien es cierto que las universidades tienen áreas de educación virtual; es verdad también que no todas teníamos la infraestructura, recursos y capacitación necesaria para hacer frente al reto en el momento de la pandemia. Y qué decir de los estudiantes, aunque poseen importantes habilidades en el manejo de las tecnologías de la información; tuvieron que enfrentar el acceso de equipo de cómputo o teléfonos inteligentes, de acceso a las redes, de manejo de plataformas o aplicaciones sin previa capacitación, y responder a las exigencias de los docentes para concluir el ciclo escolar.

Tras la suspensión de clases establecida del 23 de marzo al 17 de abril, decreto publicado en el Diario Oficial de la Federación el día 16 de marzo de 2020. Las Instituciones de Educación Superior buscaron la continuidad del ciclo escolar primero con un receso que permitía unir con el calendario escolar las vacaciones de semana santa y que en muchos de los casos permitió planear estrategias sobre la capacitación de los docentes, el manejo administrativo institucional y la segunda la habilitación de plataformas para dar continuidad al ciclo escolar. Hasta el día 30 de mayo, por decreto en el Diario Oficial de la Federación se confirmó continuar la educación virtual hasta el cierre del ciclo escolar.

Ante la inesperada suspensión de actividades académicas de manera presencial, algunos estudiantes regresaron a sus lugares de origen con implicaciones importantes sobre el acceso a dispositivos electrónicos y carencias de conectividad; las autoridades universitarias en apego a las recomendaciones emitidas en el ámbito internacional por la UNESCO (2020) y ANUIES (2020) nos llevaron al mundo de los medios digitales para continuar la labor. Este cambio fue rápido, improvisado y obligatorio.

La continuidad del trabajo académico tuvo como principal apoyo las tecnologías de la información y comunicación, y es necesario reconocer que no todos los estudiantes y docentes contaban con equipo, conectividad y capacitación suficiente para el uso y manejo de las plataformas digitales.

Según el INEGI (2020) un número significativo de alumnos cuentan con recursos tecnológicos, lo cierto es que muchos de ellos no tienen los medios necesarios para dicha transición. Según la Encuesta Nacional sobre Disponibilidad y Uso de Tecnologías de la Información en los Hogares 2019, sólo el $44.3 \%$ de la población dispone de computadora y el $70.1 \%$ de acceso a internet; no obstante, estos datos varían entre los distintos niveles socioeconómicos.

Es posible que en casa se cuente con una sola computadora que comparten los miembros de la familia y que muchos de los estudiantes no cuenten con un espacio para sus labores escolares. También es cierto que, al estar en casa, el tiempo de que disponen se divide en diversas actividades, tareas propias del hogar, tareas de cuidados de otros miembros de la familia, trabajo formal y actividades escolares. A esto se suma que existe la creencia de que estos alumnos pertenecen a la generación tecnológica, aunque muchos de ellos carecen de las habilidades necesarias para desarrollar actividades de aprendizaje de forma virtual, así como de la autodisciplina que demandan estas modalidades, en un ambiente de estrés, incertidumbre y de restricciones socioeconómicas derivadas de la pandemia

López y Rodríguez (2020) plantean tres elementos fundamentales en el análisis de la trayectoria escolar. El primero de ellos se refiere a la desigualdad educativa en relación con poder dar seguimiento a los cursos con los recursos e infraestructura que tengo en casa, con el paso del tiempo podemos apreciar las diferencias entre aquellos que viven en áreas urbanas o rurales, o por estatus socioeconómico. En segundo lugar, podemos pensar en la importante labor que realizan los docentes, quienes han tenido que planear, diseñar y alimentar diferentes plataformas digitales haciendo uso de herramientas educativas. En este contexto también encontramos grandes diferencias digitales, que en menor medida se deben a los recursos e infraestructuras, pero que son importantes en relación con las habilidades que los docentes tienen para desarrollar su labor en un ambiente virtual. Y finalmente, tenemos que reflexionar sobre la resiliencia, motivación y capacidad de autoaprendizaje y apropiación de contenidos por parte de los estudiantes, esto representa grandes diferencias en un ambiente presencial o virtual y es un elemento determinante en la permanencia de los estudiantes, porque sin duda algunos de ellos durante este proceso decidieron abandonar, interrumpir, incluso han decidido no continuar con su formación.

La pandemia originó cambios llevándonos a modalidades educativas que han evolucionado constantemente para adaptarse a los requerimientos de la sociedad moderna. La 
incorporación de las tecnologías de información y comunicación a la educación han hecho posible el cambio de los paradigmas de las escuelas tradicionales centradas en la enseñanza hacia la educación centrada en el aprendizaje del estudiante. Esta modalidad de estudio permite que los actores del proceso de enseñanza-aprendizaje puedan interactuar, tanto sincrónica como asincrónicamente, posibilita el acceso a los materiales formativos y actividades de evaluación desde cualquier lugar y en cualquier momento mientras exista conexión a internet, permitiendo a los estudiantes cada vez más a ser los gestores de su propio tiempo (McAnally-Salas, 2007). Para fines prácticos de esta investigación, utilizaremos los términos educación virtual y educación en línea para referirnos a esta modalidad de estudio.

En la educación a distancia, la mediación tecnológica es de suma importancia, pues hace posible la comunicación a través del uso multimedia, permitiéndonos compartir, no solo conocimientos sino aprendizajes. En esta modalidad, el centro de las actividades es el alumno y no el profesor y su fundamento es el autoaprendizaje (Martínez Uribe, 2008).

Los estudiantes que deseen participar en la educación en línea deben tener un manejo adecuado de la tecnología digital, según Ramírez, Casillas y Ojeda (2013) porque contempla el uso de dispositivos electrónicos, entornos gráficos de sistemas digitales, sistemas computacionales de productividad, administración de recursos multimedia, el uso de las plataformas de aprendizaje, referida a las plataformas informáticas de trabajo y a los programas y servicios de comunicación, socialización e intercambio de archivos, correo electrónico, blogs, servicios de web social; asimismo, es imprescindible el uso correcto de información a través de herramientas digitales relacionadas con búsquedas en bases de datos especializadas y bibliotecas digitales; así como hacer énfasis en prácticas de ciudadanía digital, que involucran a la ética y a las reglas de etiqueta dentro del uso de la tecnología digital (Ramírez, Casillas y Ojeda, 2013).

Después de estos acontecimientos es importante la revisión de las condiciones en las que los estudiantes han enfrentado esta pandemia, por ello nos preguntamos ¿Cómo perciben los estudiantes sus capacidades para trabajar en línea? ¿Contaban los estudiantes con la conectividad necesaria? ¿Trabajar en línea obligó al estudiante a organizar el tiempo de forma diferente? ¿Qué dificultad percibe el estudiante una vez concluido el ciclo escolar? ¿De ser necesario, el estudiante continuaría el próximo semestre su formación en línea?

El presente estudio constituye un resultado parcial del proyecto de investigación "Continuidad pedagógica en Educación Superior en tiempo de la pandemia", pretende responder las preguntas de investigación en estudiantes y docentes.

\section{METODOLOGÍA}

Para el presente trabajo se diseñó un estudio descriptivo y retrospectivo (Hernández, Fernández y Baptista, 2014) durante la última semana del ciclo escolar enero-junio 2019. La muestra estuvo conformada por 129 estudiantes universitarios, $32.6 \%$ (42) del sexo masculino y $67.4 \%$ (87) del sexo femenino de cuarto semestre de nivel Licenciatura (ver Tabla 1). Se utilizó como principal instrumento de investigación una encuesta diseñada para este propósito y distribuida vía electrónica para obtener información que sirviera para caracterizar su punto de vista sobre su capacidad para trabajar en línea, disponibilidad de equipo y conectividad, la organización del tiempo, la dificultad que representó este trabajo y su disposición para continuar el próximo semestre en línea, entre otros.

La encuesta fue aplicada de manera individual, respetando el anonimato con identificación oculta con el fin de garantizar la personalización de los resultados obtenidos y al mismo tiempo mayor objetividad en la información. Se usó el paquete SPSS en su versión 20 para el procesamiento de la información.

\section{RESULTADOS}

Los resultados obtenidos muestran que la edad promedio de los estudiantes fue $21.01+4.7$, los que contaron con conexión a internet suficiente y de calidad para realizar el trabajo en la plataforma fue el $29.5 \%$, el $53.5 \%$ con conexión regular y el $17 \%$ se reportó sin conexión. El $80 \%$ de los estudiantes tuvieron que adaptar un espacio durante la contingencia para desarrollar sus actividades académicas.

Tabla 1. Distribución de los estudiantes en estudio

\begin{tabular}{cccc}
\hline Sexo & Edad & Frecuencia & Porcentaje \\
\hline Femenino & $18-21$ & 32 & 76.2 \\
& $22-25$ & 7 & 16.7 \\
& 26 o mayor & 3 & 7.1 \\
Masculino & $18-21$ & 73 & 83.9 \\
& $22-25$ & 10 & 11.5 \\
& 26 o mayor & 4 & 4.6 \\
\hline
\end{tabular}

Nota: Elaboración propia.

Los estudiantes expresaron que al inicio de la contingencia el $40.4 \%$ no estaba preparado para trabajar de manera virtual, el $44.2 \%$ respondió más o menos preparados y el 
$15.4 \%$ se sentía totalmente preparado para este cambio. El $41.1 \%$ dijo que este proceso de continuidad no fue fácil, para el $45.7 \%$ resultó bastante fácil y para el $13.2 \%$ fue totalmente fácil.

Uno de los problemas que se pudo identificar fue que los estudiantes dejan las tareas académicas para el último momento, esto ocurrió en el $30.2 \%$ de manera regular y en el $26.3 \%$ todo el tiempo.

Fue fácil en el $44.2 \%$ trabajar con la plataforma que ofreció la universidad para concluir el ciclo escolar, el resto reportó dificultades en algún grado. Uno de los elementos que hizo falta fue la capacitación o apoyo técnico, los estudiantes expresaron en el $65.2 \%$ de los casos.

Sobre los materiales didácticos proporcionados y el tiempo que tuvieron para realizar sus actividades, los estudiantes consideraron que el $14.8 \%$ fueron poco adecuados, el $38.8 \%$ regulares y en cuanto al tiempo empleado el $45.7 \%$ lo considero exagerado. El manejo del tiempo fue un tema fundamental en la pandemia, los estudiantes reportaron que tuvieron que emplear tiempo extra para realizar las actividades en la plataforma en el 79\% de los casos. El 62.1\% de los estudiantes reconoce que les hizo falta organizar mejor su tiempo para poder responder al trabajo virtual.

En relación con la evaluación, los estudiantes manifiestan que esta fue complicada en el $76 \%$ de los casos, el trabajo fue excesivo en el $85.3 \%$ de las veces y el $53.6 \%$ piensa que la calificación del curso no corresponde al trabajo y dedicación que tuvieron, finalmente los estudiantes dijeron en el 78.3\% que su calificación no representa el conocimiento adquirido en el periodo.

Sobre las habilidades que resultaron difíciles de desarrollar en línea los estudiantes señalan: el $76 \%$ pudieron desarrollar habilidades para resolver problemas, $75.9 \%$ pensamiento crítico, $65.2 \%$ habilidades de escritura, el $84.5 \%$ desarrollo habilidades de investigación, el 64.4\% logró realizar trabajos en equipo y el $82.2 \%$ desarrolló habilidades matemáticas (Ver Figura 1).

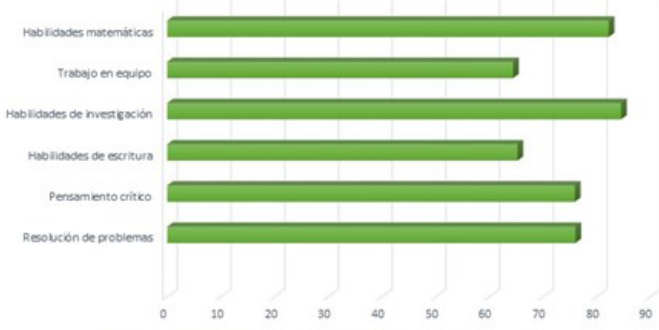

Figura 1. Habilidades desarrolladas en línea

Nota: Elaboración Propia.
La mayor parte del tiempo el $87.5 \%$ de los estudiantes se comunicaron con los docentes y sus compañeros fuera de la plataforma. Entre los medios más utilizados para comunicarse con los docentes y compañeros además de la plataforma, se destacó el WhatsApp en el 90.7\% de los casos, email $55.8 \%$, llamada telefónica 35.7\%, Google 34\%, Facebook $30.2 \%$; los menos utilizados fueron: mensaje de texto, Hangouts, Zoom, Meet (Figura 2). Estos resultados nos hacen reflexionar que, aunque tenemos a disposición múltiples medios innovadores, no hemos dejado atrás la llamada telefónica, el email y en menor proporción el mensaje de texto.

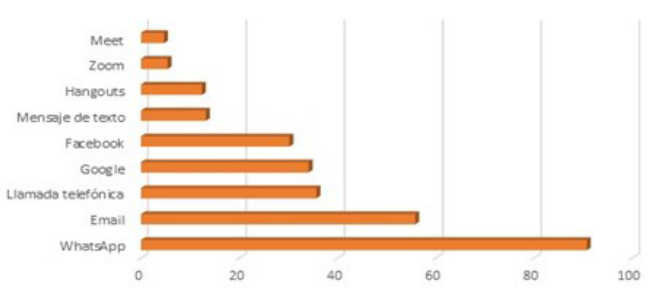

Figura 2. Medios utilizados para comunicarse con docentes y compañeros

Nota: Elaboración Propia.

Las actividades realizadas en línea fueron reportadas como poco motivadoras por el $24.8 \%$ y el estudiante piensa que la actitud del profesor fue la mejor que pudo dar dadas las circunstancias en el 59\%.

El 57.4\% de los estudiantes coincide en que representó para ellos mayores dificultades terminar sus cursos en línea. El $87.6 \%$ de los estudiantes dijeron que continuar sus estudios en línea les causo estrés. El 64.4\% reconoció hacerle falta disposición para trabajar en línea. De continuar la educación en línea, el $65.2 \%$ preferiría tomar un descanso.

Encontramos que las mujeres sienten que requieren una mejor organización, tuvieron mayor necesidad de adaptar un espacio para trabajar, se sintieron más estresadas en este proceso y tienen menos motivación por el trabajo en línea que los varones; sin embargo, estas diferencias no fueron estadísticamente significativas.

Finalmente, los resultados que muestran diferencias significativas por sexo son: que las mujeres percibieron una mayor facilidad en tomar clases en línea que los varones $(\mathrm{x} 2=11.302 . \mathrm{p}=0.023)$ y los varones en mayor proporción para continuar sus estudios, prefieren esperar a que todo se normalice $(\mathrm{x} 2=10.785, \mathrm{p}=0.029)$.

\section{DISCUSIÓN}

Los resultados obtenidos permitieron describir como los estudiantes perciben el trabajo en línea, encontrando que para ellos el tránsito de un ambiente presencial a uno virtual 
fue difícil, especialmente para los varones. Pero no podemos negar que el principal determinante fue la conectividad porque un importante número de estudiantes no contaban con acceso a internet de calidad para llevar a cabo las tareas, en coincidencia con lo reportado en la Encuesta Nacional de Disponibilidad de las Tecnologías de la Información en los hogares 2019, realizada por el INEGI, que muestra que el $56.4 \%$ de los hogares tienen conexión a internet en México.

La literatura reporta que la enseñanza presencial o en línea no muestra diferencias respecto a su efectividad, algunos autores han encontrado que la enseñanza virtual puede obtener inclusive mejores resultados (Siemens, Gaševi囚 y Dawson, 2015) (Allen y Seaman, 2017). Por ello, debemos reconocer que el rendimiento de los estudiantes depende más de los diseños pedagógicos de cada acción formativa que de los recursos seleccionados para el aprendizaje. Sin embargo y dado el contexto encontramos resistencia y dificultades en el ambiente virtual, que puede ser atribuido a las circunstancias en las que se realiza el cambio; poca planeación, organización y la obligatoriedad de concluir de esta manera.

Frecuentemente en la evaluación en línea se observa una tendencia hacia formas tradicionales de evaluación (exámenes vigilados y tareas escritas), así como pocas oportunidades para la variedad en las evaluaciones y limitado desarrollo de habilidades genéricas tales como habilidades comunicacionales, capacidades tecnológicas para la lectura y escritura, solución de problemas, trabajo en equipo, y otras similares. (Dorrego, 2006)

Los estudiantes manifestaron haber desarrollado algunas de las habilidades antes mencionadas al menos en la mitad de ellos, sin embargo, también reportan que la evaluación fue complicada en su mayoría y que la calificación no corresponde al trabajo y dedicación realizada. Para superar esa limitación Dorrego (2006) expone que la evaluación en línea debe diseñarse y desarrollarse tomando en cuenta las características de los aprendizajes derivadas de sus fundamentos: constructivista, basado en recursos, colaborativo, basado en problemas, situado, entre otras.

Podemos concluir que el tránsito de un ambiente presencial a uno virtual fue difícil para los estudiantes, un grupo de estos no contó con conexión a internet para realizar el trabajo en la plataforma, poco menos de la mitad de los estudiantes no se sentía preparado para trabajar de manera virtual, e implico un mayor esfuerzo y dedicación. 


\section{REFERENCIAS}

Allen, E. y Seama, J. (2017). Digital Learning Compass: Distance Education Enrollment Report 2017. Babson Survey Research Group e-Literate, and WCET.

ANUIES (2020), "Acuerdo Nacional por la Unidad en la Educación Superior frente a la emergencia sanitaria provocada por el covid-19", 24 de abril, consultado el 12 de mayo, 2020.

Dorrego, E. (2006). Educación a Distancia y Evaluación del Aprendizaje. RED. Revista de Educación a Distancia, número M6 (Número especial dedicado a la evaluación en entornos virtuales de aprendizaje). Consultado (24/06/2020) en http://www.um.es/ ead/red/M6

Hernández Sampieri, R., Fernández Collado, C., y Baptista Lucio, P. (2014). Metodología de la investigación: Roberto Hernández Sampieri, Carlos Fernández Collado y Pilar Baptista Lucio (6a. ed. --.). México D.F.: McGraw-Hill.

INEGI (2020), "Encuesta Nacional sobre Disponibilidad y Uso de Tecnologías de la Información en los Hogares 2019", México

López Ramírez M. y Rodríguez S. A., (2020) Trayectorias escolares en la educación superior ante la pandemia ¿continuar, interrumpir o desistir? En Educación y pandemia. Una visión académica, México, UNAM, IISUE Pág. 103-108

http://www.iisue.unam.mx/nosotros/covid/educacion-y-pandemia

Martínez Uribe Carmen Heedy (2008) La educación a distancia: sus características y necesidad en la educación actual. Educación Vol. XVII, núm. 33: 7-27.

McAnally-Salas, L. (2007). Factores contextuales y de formación del docente que influyen en el diseño de cursos en línea. Tesis doctoral. Recuperado el 15 de febrero de 2013, de http://iide.ens.uabc.mx/blogs/ mcanally/files- /2007/12/tesis-doctoral-mcanally. pdf

Ramírez, A., Casillas, M. A., y Ojeda Ramírez, M. M. (2013). Brecha Digital entre estudiantes y profesores de la universidad Veracruzana: grado de apropiación tecnológica, capital cultural, trayectorias escolares y desempeño académico. Recuperado el 10 de enero de 2015, de Recuperado el 30 de octubre de 2020, de http://www.uv.mx/blogs/brechadigital/ files/2013/08/brecha_digital_noviembre_2013.zip

Siemens, G., Gaševic, D., y Dawson, S. (Ed.), (2015). Preparing for the digital university: a review of the history and current state of distance, blended, and online learning. Edmonton, AB: Athabasca University.

UNESCO (2020), "El coronavirus covid-19 y la educación superior: impacto y recomendaciones", 2 abril, consultado el 4 de mayo, 2020. 


\section{ANEXO 1}

\section{ESTRUCTURA DE LA ENCUESTA}

UNIVERSIDAD AUTÓNOMA DE CHIHUAHUA

FACULTAD DE CIENCIAS DE LA CULTURA FÍSICA

PROYECTO: CONTINUIDAD PEDAGÓGICA EN EDUCACIÓN SUPERIOR EN TIEMPOS DE

\section{LA PANDEMIA}

\begin{tabular}{|l|}
\hline Edad \\
\hline Sexo \\
\hline Correo electrónico \\
\hline
\end{tabular}

Se preguntó a los estudiantes sobre la siguiente temática:

\begin{tabular}{|l|}
\hline Equipo de cómputo en casa \\
\hline Equipo de cómputo personal \\
\hline Conexión a internet suficiente y de calidad \\
\hline Dificultad de clases en línea \\
\hline Organización del tiempo \\
\hline Uso de la plataforma \\
\hline Necesidad de capacitación para tomar clases en línea \\
\hline Necesidad de apoyo técnico \\
\hline Adaptación de espacios para trabajar \\
\hline Materiales didácticos \\
\hline La evaluación en línea \\
\hline El desarrollo de competencias \\
\hline Medios de comunicación adicionales a la plataforma \\
\hline Implicaciones en tiempo y dificultad del trabajo en \\
línea \\
\hline
\end{tabular}

